\title{
Extracellular vesicle-mediated transfer of miR-21-5p from mesenchymal stromal cells to neurons alleviates early brain injury to improve cognitive function via the PTEN/Akt pathway after subarachnoid hemorrhage
}

\author{
Xiao Gao ${ }^{1,2,3}$, Ye Xiong ${ }^{1,4}$, Qizhao Li ${ }^{1,5}$, Min Han', Dezhi Shan', Guozheng Yang ${ }^{1}$, Shouji Zhang ${ }^{1}$, Danqing Xin ${ }^{5}$, \\ Rongrong Zhao ${ }^{1,2}$, Zhen Wang ${ }^{5}$, Hao Xue ${ }^{1,2}$ and Gang Li, ${ }^{1,2,3}$
}

\begin{abstract}
Patients with subarachnoid hemorrhage (SAH) often suffer from cognitive function impairments even when they have received proper treatment, such as the clipping or coiling of aneurysms, and this causes problems with returning to work and burdens the family. Increasing attention has been paid to mesenchymal stem cell (MSC)-derived extracellular vesicle (MSC-EV) as promising therapeutic vesicles for stroke management. In this study, we explored the potential role of MSC-EV in a rat model of SAH. We observed that MSC-EV ameliorated early brain injury (EBI) after SAH by reducing the apoptosis of neurons and that SAH induced an increase in the expression level of miR-21 in the prefrontal cortex and hippocampus. In addition, using miRNA profiling and CSF sequencing data from the exRNA Atlas, we demonstrated that EV-derived miR-21 protected neurons from apoptosis and alleviated SAH-induced cognitive dysfunction. The neuroprotective role of MSC-EV was abrogated by miR-21 knockdown or the administration of MK2206, a PTEN/Akt inhibitor. Overall, our results suggest that MSC-EV promotes neuronal survival and alleviates EBI after SAH through transferring miR-21 to recipient neurons.
\end{abstract}

\section{Introduction}

Subarachnoid hemorrhage (SAH) is a life-threatening neurological disease with high mortality and disability rates $^{1,2}$. However, those patients who survive acute bleeding and rebleeding often suffer from cognitive deficits with decreased life quality for years after $\mathrm{SAH}^{3-5}$. Increasing evidence indicates that early brain injury (EBI) may account for the poor outcomes observed in SAH patients $^{6-8}$.

\footnotetext{
Correspondence: Gang Li (ligangqiluhospital@163.com)

'Department of Neurosurgery, Qilu Hospital, Cheeloo College of Medicine,

Shandong University, 107 Wenhua Xi Road, Jinan, Shandong Province, China ${ }^{2}$ Institute of Brain and Brain-Inspired Science, Shandong University, 44 Wenhua Xi Road, Jinan, Shandong Province 250012, P.R. China

Full list of author information is available at the end of the article.

Edited by Y. Shi
}

Recently, an increasing amount of attention has been paid to mesenchymal stem cell (MSC)-derived EVs, as messengers that function in intercellular communication and promising therapeutic vesicles used for regeneration medicine, which are believed to be able to replace MSC cell therapy ${ }^{9}$. In addition, theoretically, using secreted EVs rather than cells themselves could overcome some safety problems with MSC therapy. EVs contain proteins, lipids, mRNAs, and noncoding RNAs that can modulate the pathophysiological state of receiver cells. MSC EV-based therapy has shown promise in experimental models of liver injury ${ }^{10,11}$, renal injury ${ }^{12}$, acute myocardial infarction $^{13}$, wound healing ${ }^{14}$, and so on.

MSC cell therapy has been used in the restorative process after stroke and traumatic brain injury (TBI) since the early

\section{(c) The Author(s) 2020}

(c) (i) Open Access This article is licensed under a Creative Commons Attribution 4.0 International License, which permits use, sharing, adaptation, distribution and reproduction in any medium or format, as long as you give appropriate credit to the original author(s) and the source, provide a link to the Creative Commons license, and indicate if changes were made. The images or other third party material in this article are included in the article's Creative Commons license, unless indicated otherwise in a credit line to the material. If material is not included in the article's Creative Commons license and your intended use is not permitted by statutory regulation or exceeds the permitted use, you will need to obtain permission directly from the copyright holder. To view a copy of this license, visit http://creativecommons.org/licenses/by/4.0/. 
2000s. Additionally, much evidence has suggested that neuroprotective effects are mediated by EVs secreted by MSCs. For example, Michael Chopp et al. demonstrated that MSC EVs could be taken up by neural cells. These miR-133b-enriched EVs could help restore neurological function ${ }^{15,16}$. However, only a few studies concerning the role of MSC therapy in SAH have been reported, and these have demonstrated that the neuroprotective effects partly resulted from the alleviation of microglia-mediated neuroinflammation ${ }^{17,18}$. However, whether MSC EVs play a part in SAH therapy remains unclear.

MiRNA is one of the important cargoes encapsulated in EVs. These miRNAs serve as messengers for intercellular communications, biomarkers for clinical diagnosis and prognosis, and therapeutic agents for diseases. Bache et al. reported that miRNA profile changed after $\mathrm{SAH}^{19}$, while they didn't explore the mechanisms of miRNA upregulation.

The aim of this study was to investigate the therapeutic potential of MSC EVs in SAH, especially for cognitive function recovery, and to explore the underlying mechanisms. We demonstrated that MSC EVs can alleviate $\mathrm{EBI}$ and improve cognitive function after $\mathrm{SAH}$ and that the mechanism involved mainly depends on the transfer of MSC-derived EV-derived miR-21-5p to neurons to activate AKT signaling to inhibit apoptosis.

\section{Materials and methods}

\section{MSC-EV isolation and characterization}

Bone marrow-derived MSCs were obtained from Cyagen Bioscience (Suzhou, China) and cultured with amodified MEM medium containing 10\% fetal bovine serum (FBS) and penicillin-streptomycin in $175 \mathrm{~cm}^{2}$ tissue culture flasks. For EVs isolation, we replaced the conventional culture medium with $20 \mathrm{ml}$ medium containing 10\% EV-depleted FBS (Vivacell Biotechnology, Germany) when the cells reached $60-80 \%$ confluence. Following an additional $48 \mathrm{~h}$ of culturing, the media from about $5-8 \times 10^{6}$ cells were then collected for centrifugation $^{20}$. The EVs were stored at $-80^{\circ} \mathrm{C}$.

The concentration of EVs was determined by using Enhanced BCA Protein Assay Kit (Beyotime). $40 \mathrm{ml}$ culture medium from two T175 flasks contained about $600 \mu \mathrm{g}$ of EV. To determine the size distribution of the EVs, nanoparticle tracking analysis was performed using the qNano system (Izon) on samples diluted with PBS. And antibodies of EV markers and negative controls were used for western blot analysis: TSG101 (1:1000, Cell Signaling), CD9 (1:1000, Cell Signaling) and Calnexin (1:1000, Cell Signaling).

\section{SAH rat model}

The intravascular perforation SAH model was induced as previously described ${ }^{21,22}$. Briefly, adult male SD rats weighing 300-320 g were anesthetized through an intraperitoneal injection of chloral hydrate $(340 \mathrm{mg} / \mathrm{kg}$ body weight). After the left carotid artery and associated branches were dissected, a 3-0 monofilament nylon suture was inserted into the stretched external carotid artery, then guided into the internal carotid artery wall and was withdrawn following the perforation. Rats in the sham group underwent the same surgical procedure without perforation. This study was performed in accordance with relevant guidelines and approved by the Ethics Committee of Qilu Hospital.

\section{Experimental design}

All animal experiments underwent randomization at entry. Animal subjects were randomly assigned to treatment groups and during analysis. For immunostaining imaging experiments, the experimenter was blinded to the slides selected for imaging on microscopy.

\section{Experimental design 1}

To determine the effects of MSC-EV on EBI after SAH, 48 rats (59 rats were used, but only 48 rats survived after the surgery) were randomly assigned into three groups: sham, SAH + PBS and SAH + MSC-EV $(n=16)$. All rats were sacrificed at $48 \mathrm{~h}$ after $\mathrm{SAH}$ for assays including neurological score assessment, brain water content, western blot, and histopathological observation.

\section{Experimental design 2}

Nine rats were used to evaluate the distribution of PKH67-labeled MSC-EV administered by i.v. throughout the whole body. Nine rats ( 15 rats were used, but only 9 rats survived) were randomly assigned to three groups: sham, SAH + PBS and SAH + MSC-EV $(n=3)$. Twentyfour hours after MSC-EV administration, all rats were deeply anesthetized and perfused with $4 \%$ paraformaldehyde prior to frozen sectioning.

\section{Experimental design 3}

A total of 32 rats (41 rats were used, but only 32 rats survived) were used in this cohort, in which rats were randomly divided into eight subgroups: sham, 3, 6, 12, 24, $48,72 \mathrm{~h}$ and 1 -week post-SAH $(n=4)$. Each group of rats was sacrificed at the indicated time post SAH for RNA extraction from the prefrontal cortex and hippocampus.

\section{Experimental design 4}

To explore the molecular mechanism underlying neuroprotection by MSC-EV, we employed miR-21-5p inhibitors and MK2206 to block its therapeutic effects. Fifty rats (63 rats were used, but only 50 rats survived) were randomly divided into the following groups: sham, $\mathrm{SAH}$ + PBS, SAH + MSC-EV, SAH + MSC-EV(-21) and SAH + MSC-EV + MK2206. Each group of rats was sacrificed 
at $48 \mathrm{~h}$ after SAH for neurological function, western blot, HE staining, TUNEL assay and double immunostaining of NeuN and cleaved caspase- 3 .

\section{Experimental design 5}

Thirty rats (36 rats were used, but only 30 rats survived) were used to assess cognitive function impairments after SAH. The Morris Water Maze task was performed to evaluate cognitive changes as previously described ${ }^{23}$ in all groups of rats, including the sham, SAH + PBS, and SAH + MSC-EV groups $(n=10)$.

\section{EVs tracing}

EVs were dyed with PKH67 according to the manufacturer's protocol. In brief, $500 \mu \mathrm{g}$ MSC-EVs were incubated with $4 \mu \mathrm{l}$ PKH67 diluted in $1 \mathrm{ml}$ dyeing buffer for $15 \mathrm{~min}$, then $2 \mathrm{ml}$ of stopping buffer containing $0.5 \%$ bovine serum albumin (BSA) was added, and the labeled EVs were washed by centrifugation at $100,000 \times g$ for $4 \mathrm{~h}$. The labeled EVs were resuspended with PBS before administration. As negative control, $4 \mu \mathrm{l}$ PKH26/ PKH67 dye was added to $1 \mathrm{ml}$ dyeing buffer and incubated with equivalent volume of PBS for $15 \mathrm{~min}$. And this collection containing little free dye from ultracentrifugation was injected into SAH rats as control.

\section{Neurological scoring}

At $48 \mathrm{~h}$ after $\mathrm{SAH}$, we assessed neurobehavioral function according to the modified Garcia scoring system ${ }^{24,25}$. In brief, this system included six tests: spontaneous activity ( $0-3$ points), reaction to side stroking (1-3 points) and to vibrissae touch (1-3 points), limb symmetry ( $0-3$ points), forelimb outstretching (0-3 points), and climbing (0-3 points). The total scores on these tests ranged from 3 to $18^{26,27}$. Higher scores indicated better neurological behavior.

\section{Brain water content}

The brains were quickly dissected from the skulls $48 \mathrm{~h}$ after SAH and then weighed before and after $48 \mathrm{~h}$ of heating at $95^{\circ} \mathrm{C}$. Brain edema was calculated as (wet weight - dry weight)/wet weight $\times 100 \%$.

\section{TUNEL assay}

Apoptosis was detected using an in-situ cell death detection kit (Roche) according to the manufacturer's protocol. After counterstaining with DAPI, the slides were kept in Antifade Mounting Medium (Beyotime). Three random microscope fields $(\times 20)$ were imaged for every slide of brain tissue.

\section{RNA extraction and qRT-PCR}

Total RNA was extracted from the prefrontal cortex and hippocampus using TRIzol (Invitrogen) according to the manufacturer's instructions. $1 \mu \mathrm{g}$ RNA was reversetranscribed into cDNA using the ReverTra Ace qRTPCR kit (Toyobo). Real-time PCR was performed using the SYBR Premix MASTER Kit (Roche) with a LightCycler 480 Instrument (Roche). All data for each sample were collected in triplicate. Standard curves were generated, and the relative amount of miRNA was normalized to the amount of U6 $\left(2^{-\Delta \Delta C t}\right)$.

\section{Western blot analysis}

Western blot analysis was performed as described previously $^{23}$. The protein concentration was determined using an Enhanced BCA Protein Assay Kit (Beyotime). Protein samples $(30-50 \mu \mathrm{g})$ were loaded onto $10 \%$ or $12 \%$ SDS-polyacrylamide gel for electrophoresis. Then polyvinylidene difluoride membranes (Millipore) were used for protein transfer and incubated with the following primary antibodies: Bax (1:2000, Abcam), Bcl-2 (1:1000, Abcam), cleaved caspase-3 (1:500, Cell Signaling), phospho-Akt (Ser473) (1:500, Cell Signaling), Akt (1:1000; Cell Signaling), and phosphatase and tensin homolog deleted on chromosome 10 (PTEN) (1:500, Cell Signaling). GAPDH (1:2000, Sigma-Aldrich) was used as an internal control. Horseradish peroxidase conjugated to either goat anti-mouse or rabbit IgG was used as a secondary antibody (1:5000, Cell Signaling). The membranes were detected using ChemiDoc XRS+ (Bio-Rad).

\section{Immunofluorescence imaging}

The slides were fixed in $4 \%$ paraformaldehyde for $15 \mathrm{~min}$ and blocked with $10 \%$ goat serum in PBS. The slides were incubated overnight in a humidified chamber at $4{ }^{\circ} \mathrm{C}$ with the following primary antibodies: NeuN, 1:500, Abcam; cleaved caspase-3, 1:100, Cell Signaling. After primary antibody incubation, the samples were washed with PBS and incubated with the matching fluorescent-conjugated secondary antibody (1:500 dilution, Thermo Fisher) at room temperature for $1 \mathrm{~h}$. Images were captured using a Leica DMi8 microscope. Three microscope fields $(\times 20)$ showing active caspase-3/NeuN double-positive cells were chosen and imaged. The number of active caspase-3/NeuN double-positive cells was calculated as the mean of the numbers of cells counted in six images from each rat. Four rats were included for the staining of each group. Counting was performed in a blinded manner.

\section{Fluorescence in situ hybridization (FISH)}

Rno-miR-21-5p probe (sequences: 5'-TCAACATCAG TCTGATAAGCTA-3') was synthesized by GenePharma (Shanghai, China) and used under the instructions of miRNA FISH kit (GenePharma). Briefly, On day 1, the frozen sections were rehydrated and digested by proteinase $\mathrm{K}$ for $20 \mathrm{~min}$ at $37^{\circ} \mathrm{C}$. Next, sections underwent 
pretreatment with denaturing solution for $8 \mathrm{~min}$ at $78^{\circ} \mathrm{C}$. The sections were then incubated with miR-21-5p-5p probe overnight at $37^{\circ} \mathrm{C}$. On day 2 , the sections were rinsed in pre-warmed washing solution at $43^{\circ} \mathrm{C}$ and PBS after hybridization. Thereafter, sections were blocked using $5 \%$ bovine serum albumin (BSA) for $60 \mathrm{~min}$ at $37^{\circ} \mathrm{C}$ and incubated overnight at $4{ }^{\circ} \mathrm{C}$ with NeuN antibody. On day 3 , sections were incubated with the fluorescent secondary antibody for $1 \mathrm{~h}$ at room temperature in a dark room. The nuclei were counterstained with DAPI.

\section{Intracerebroventricular drug administration}

Briefly, rats were placed in a stereotaxic apparatus under inhalation anesthesia. $100 \mu \mathrm{g}$ MK2206 in $5 \mu \mathrm{l}$ DMSO was slowly injected into left ventricle with a $10-\mu$ l Hamilton syringe (Hamilton, USA) through a drilling hole according to the following coordinates relative to bregma: $1.5 \mathrm{~mm}$ posterior and $1.0 \mathrm{~mm}$ lateral and $3.5 \mathrm{~mm}$ deep.

\section{Statistical analysis}

SPSS 20 and GraphPad Prism were used for the statistical analysis and graph plotting, respectively. The neurological scores by modified Garcia scoring system were analyzed by Kruskal-Wallis one-way analysis of variance (ANOVA) and Dunn's post hoc test. In addition, the test data are presented as the mean \pm SD and analyzed by oneway ANOVA followed by Tukey's post hoc analysis for multiple comparisons of means. When $p<0.05$, the difference was considered statistically significant.

\section{Results}

\section{MSC-EV attenuated neurological deficits after SAH}

MSC-EV was isolated from FBS-depleted culturing medium of MSC and characterized by qNano and western blotting of EV protein markers (Fig. S1). To determine the effects of MSC-EV, $100 \mu \mathrm{g}$ of EVs were slowly injected into the caudal vein of each SAH rat $1 \mathrm{~h}$ post bleeding, while the control group rats were administered an equivalent volume of phosphate-buffered saline (PBS). Compared with the SAH + PBS group, the SAH + EV group achieved higher neurological scores at $48 \mathrm{~h}$ after SAH insult, implying improved neurological performance (Fig. 1c). The brain water content also decreased after MSC-EV administration, as shown in Fig. 1d. Brain injury was also determined by HE staining (Fig. 1a) and Nissl staining (Fig. 1b). Many more neurons exhibited a contracted appearance in the SAH group, while MSC-EV alleviated this type of damage.

Moreover, the Morris Water Maze (MWM) test was performed to evaluate cognitive functional impairment in a relatively long term after SAH. Two weeks after perforation, a four-day hidden platform test and a one-day probe test were performed successively. Compared with the sham group, the SAH group showed obviously impaired cognitive function and learned more slowly, while MSC-EV improved cognitive behavior and shortened the time spent seeking the hidden island (Fig. 1f). Furthermore, in the probe test, SAH rats treated with MSC-EV entered the target quadrant more often and stayed in the island zone longer than SAH rats treated with PBS (Fig. $1 \mathrm{~g}$ and $\mathrm{h}$ ), indicating that a stronger memory was obtained.

\section{MSC-EV inhibited neuronal apoptosis resulting from EBI}

To investigate the protective mechanism of MSC-EV, we performed EV tracing assays using PKH67-dyed EVs. After sacrificing the animals, brain sections were obtained and further stained with neuronal or astrocytic markers to determine which types of cells took up the EVs. As shown in Figs. 2a, b and S3, most EVs were taken up by neurons, while little free dye couldn't target damaged brain region in control group (Fig. S2). In addition, PKH67-dyed EVs were also found in the liver, kidney, and spleen of SAH rats (Fig. S4). Meanwhile, the number of TUNEL-positive neurons was increased $48 \mathrm{~h}$ after $\mathrm{SAH}$, and the number of apoptotic cells in the cortex and hippocampus were reduced significantly after treatment with MSC-EV (Figs. 2c, d and S5). Caspase 3/NeuN staining was also used to verify SAH-induced neuronal apoptosis. As shown in Fig. $3 \mathrm{a}$ and $\mathrm{b}, \mathrm{MSC}-\mathrm{EV}$ administration significantly inhibited neuronal apoptosis after SAH. In addition, the protective role of MSC-EV was demonstrated by western blot (Fig. $3 e)$. The expression of Bcl-2 increased as a result of the effects of MSC-EV, while the expression of Bax and cleaved-caspase-3 decreased compared with that in the SAH-PBS group. Consistent with the results of our previous studies, PSD95 and PTEN were upregulated after SAH onset ${ }^{23,28}$, and MSC-EV alleviated the pathological accumulation of both targets. PSD95 participates in the formation of synapses, while its role in SAH still remains unclear.

\section{miRNA sequencing showed potential key targets in MSC- derived EVs}

MicroRNA is one of the most prominent types of content in EVs and plays an important role in EVmediated intercellular communication. Because of this, we used high-throughput sequencing to identify miRNA species and their abundance in SD-MSC-derived EVs. According to the sequencing data, miRNA makes up above half of total non-coding RNA in EVs (Fig. 4a), $55.47 \%$ of which is in single-chain mature form (Fig. 4b). As shown in Fig. 4c and d, miR-21-5p, along with the other top 6 miRNAs in terms of abundance, constitute nearly $60 \%$ of the miRNA contents in EVs. And miR-21$5 \mathrm{p}$ alone accounts for as much as $22.5 \%$ of contents. In the meantime, the let7 miRNA family drew much attention from us, with the four of let-7i, let-7c, let-7f, and let-7b 


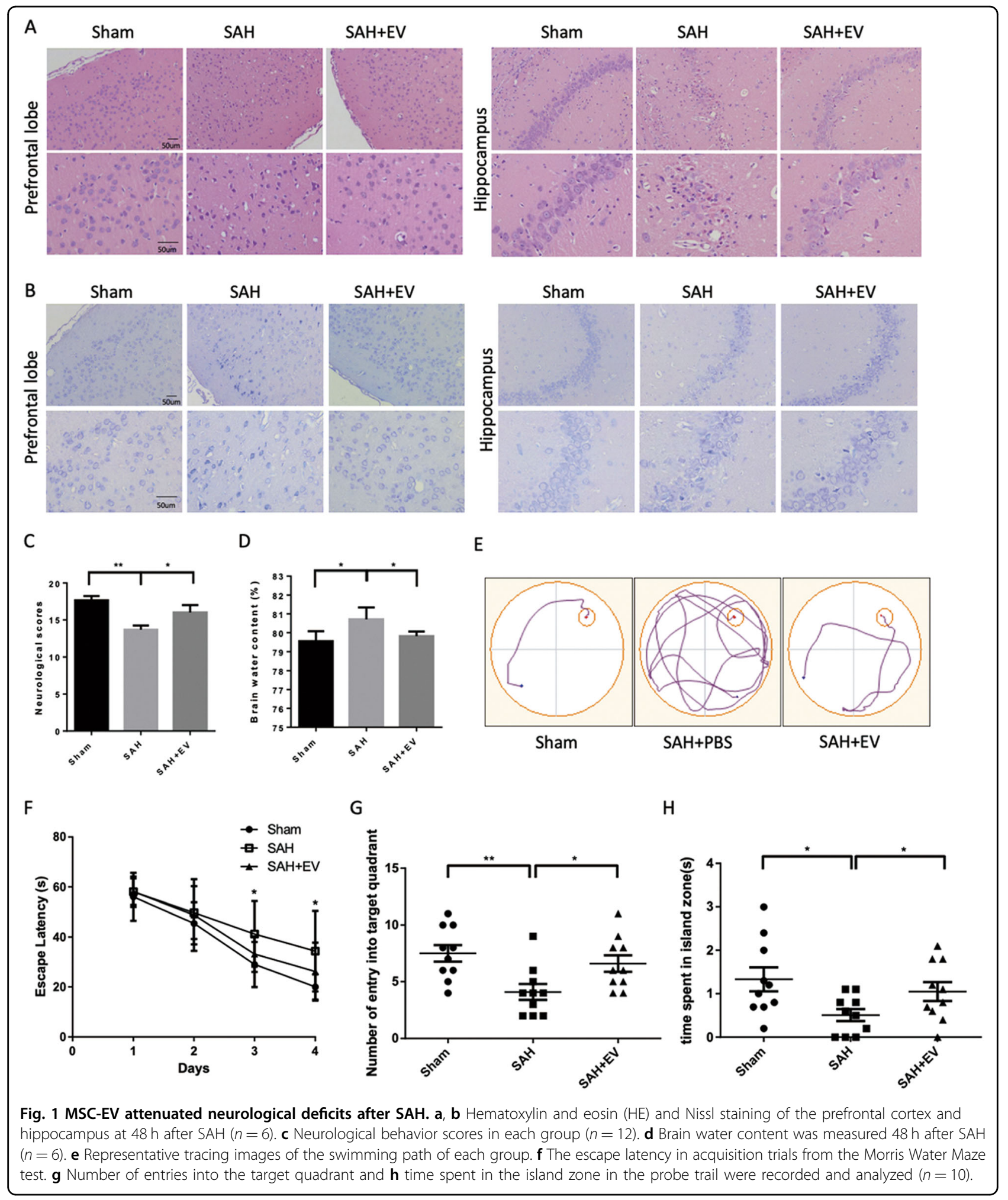

lied in top 7 miRNAs. However, according to sequencing data (5UH2TR000891-02) derived from SAH patient cerebrospinal fluid (CSF) from the exRNA Atlas ${ }^{29}$,
miR-21-5p expression increased significantly after SAH compared with that found in healthy donors (Fig. 5a, b, and S6), while the expression of let-7 family didn't exhibit 

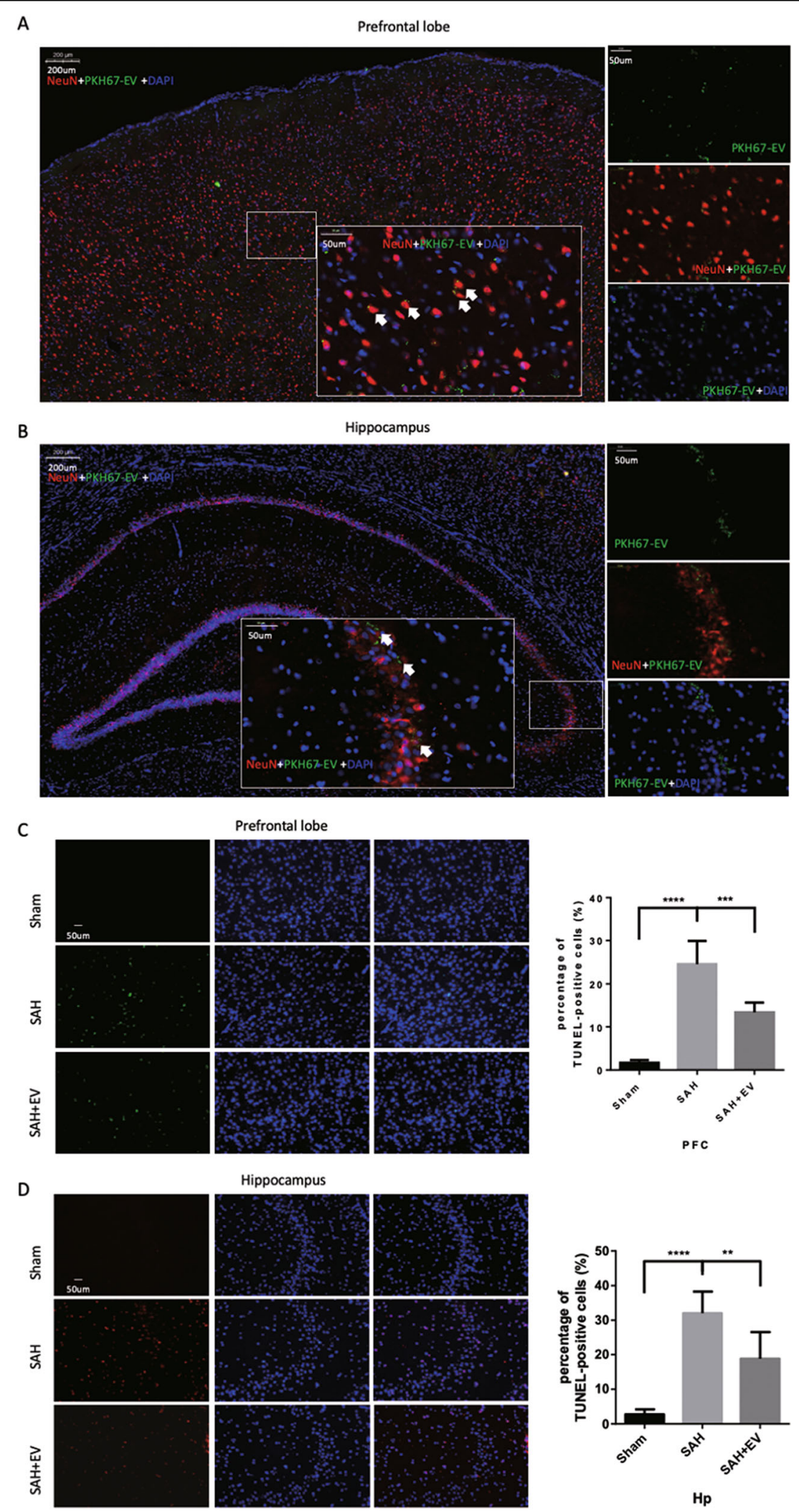

$\mathrm{Hp}$

Fig. 2 MSC-EV target injured neurons and inhibit apoptosis after SAH. $\mathbf{a}, \mathbf{b}$ Representative fluorescence images of brain sections stained with neuronal (NeuN) marker $48 \mathrm{~h}$ after SAH. PKH67-dyed EVs were administered by i.v. into SAH-affected rats $(n=3)$. c , d TUNEL staining of the cerebral cortex and hippocampus, respectively. 


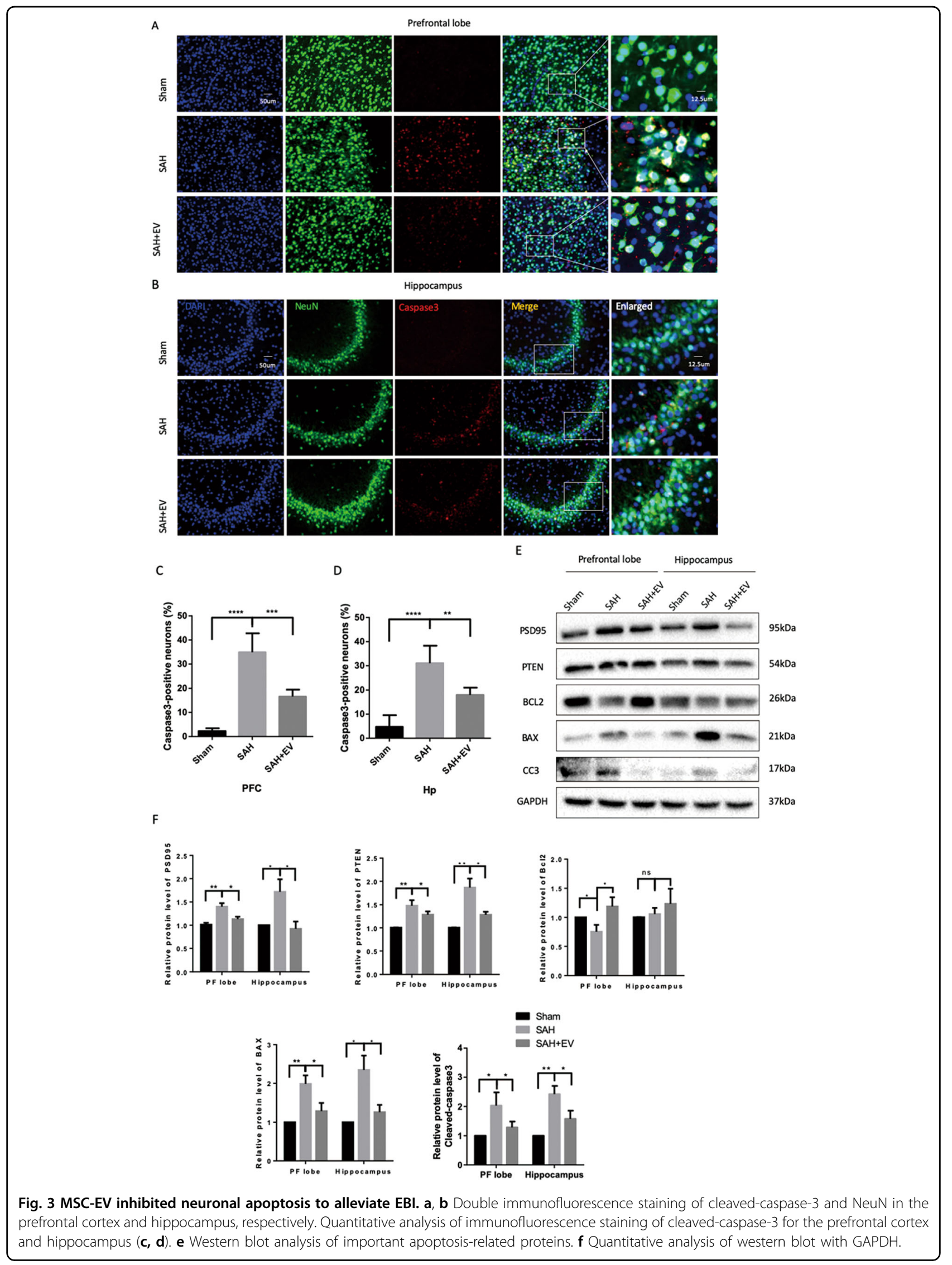




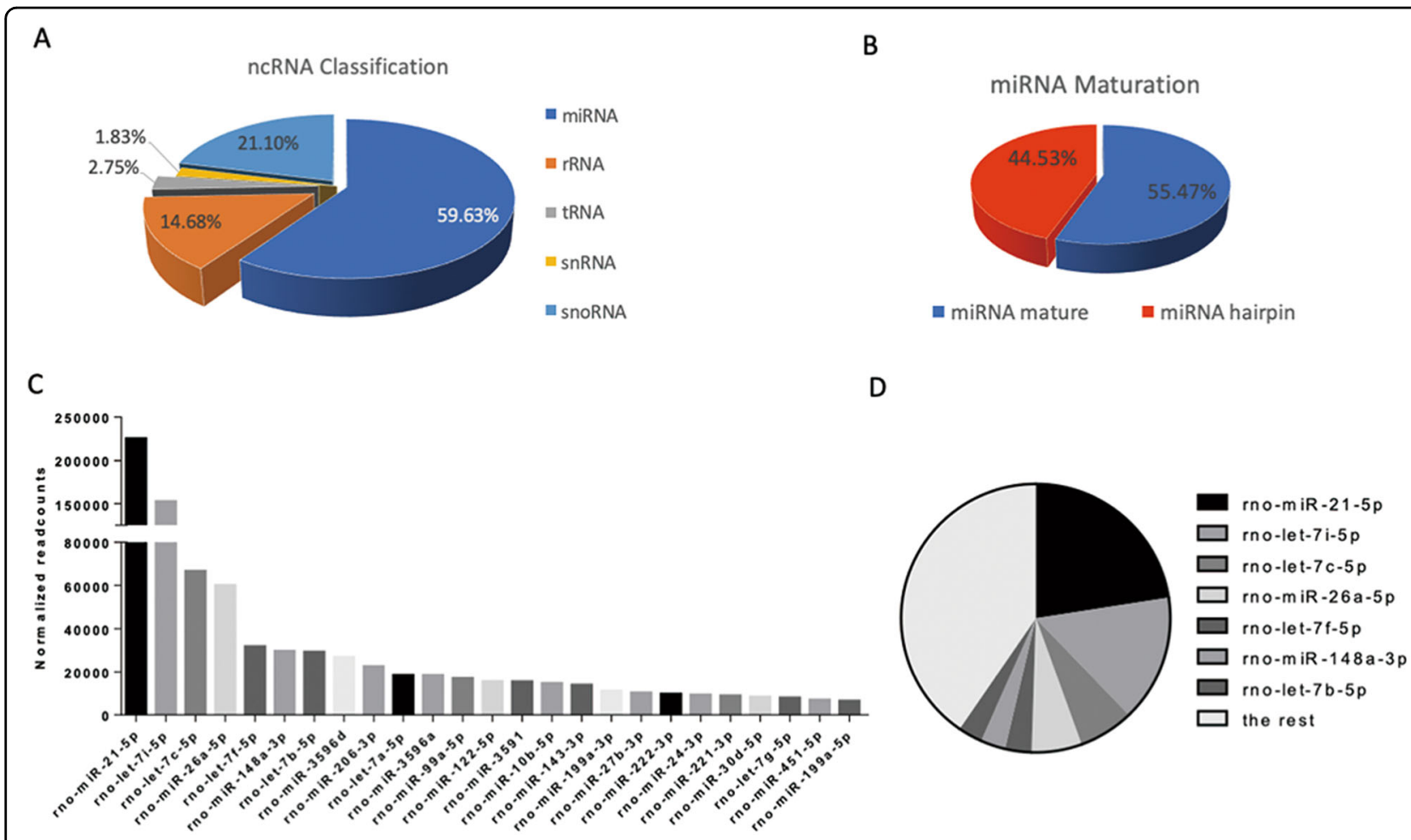

Fig. 4 miRNA sequencing revealed potential key targets in MSC-derived EVs. a Non-coding RNA sequencing revealed microRNA is the largest portion. $\mathbf{b}$ miRNA maturation analysis indicated that about half miRNA was single-chain mature form. $\mathbf{c}$ Total numbers of reads representing the top 25 miRNAs according to miRNA profiling of MSC-derived EVs are shown. $\mathbf{d}$ The top 7 miRNAs accounted for 59.6\% of the total miRNA in MSC EVs.

consistency. We also performed qRT-PCR to determine the expression of other miRNAs in prefrontal cortex and hippocampus $48 \mathrm{~h}$ post SAH (Fig. S7). Considering the above, we supposed that miR-21-5p may mediate the neuroprotective role of MSC-EV.

\section{SAH induced miR-21-5p upregulation}

Dozens of miRNAs have been shown to be critical for the development of neurological diseases ${ }^{30}$, among which miR-21-5p has been extensively studied ${ }^{31-34}$.

PCR was performed to assess the time course of miR21-5p expression after SAH. As shown in Fig. 5c, the expression level of miR-21-5p increased and peaked at $3 \mathrm{~h}$ post SAH. However, it was decreased at $3 \mathrm{~h}$ and increased at $12 \mathrm{~h}$ in the hippocampus (Fig. 5d). The expression and distribution of miR-21-5p was further identified by FISH $48 \mathrm{~h}$ after SAH. As shown in Fig. 5e, compared with the sham group, miR-21-5p expression was increased in the cortex $48 \mathrm{~h}$ after SAH in the SAH group. In addition, the increases in miR-21-5p were mostly observed in NeuNpositive cells (Fig. 5e). Thus, we assume that miR-21-5p expression was upregulated and played a protective role in neurons after SAH but decreased somehow over time, while MSC-EV administration could reverse the loss of miR-21-5p to inhibit damage.
MiR-21-5p mediated the neuroprotective effects of MSC-EV

First, we detected the expression of assumed-protective miR-21-5p. In addition, miR-21-5p expression was examined by qRT-PCR at $48 \mathrm{~h}$ after SAH. As shown in Fig. $6 \mathrm{a}$ and b, miR-21-5p expression in the prefrontal cortex increased significantly, while its expression in the hippocampus decreased without significance at $48 \mathrm{~h}$ after SAH. As expected, during treatment with MSC-EV, miR-21-5p expression increased significantly, implying that the neuroprotective effects of MSC-EVo were mediated by miR-21-5p.

To verify the importance of miR-21-5p, we transfected synthesized miR-21-5p inhibitors in MSCs to block miR21-5p activity in EVs. The blocking effect was detected by a TUNEL assay (Fig. 6c) and caspase3/NeuN staining (Fig. 6d). The MSC-EV(-21) failed to inhibit neuronal apoptosis caused by $\mathrm{SAH}$. In addition, western blot results also showed that miR-21-5p inhibitors effectively suppressed the anti-apoptotic effects of MSC-EV on neurons after SAH (Fig. 6g). The PTEN/PI3K-AKT signaling pathway has been widely studied as a downstream of miR-21-5p in many pathophysiological processes. In the context of $\mathrm{SAH}$, we discovered that PTEN expression was upregulated and suppressed by EV-derived miR-21-5p from cultured MSCs (Fig. 6g). 


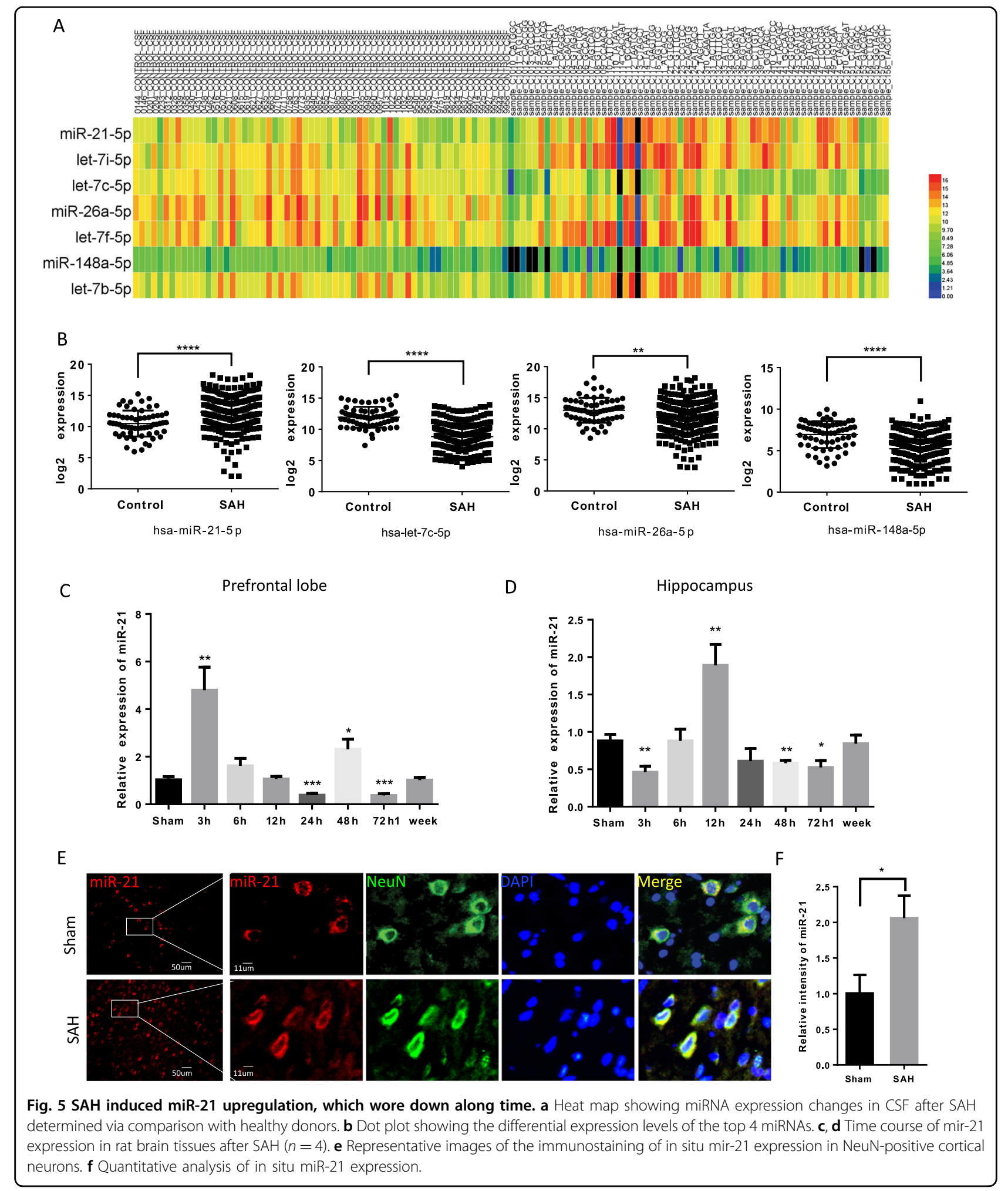

As previously mentioned, we speculated that miR-21-5p in neurons wore down through EV excretion. Thus, we examined the expression of miR-21-5p in cultured rat neurons and their conditioned medium (Fig. $7 \mathrm{a}$ and $\mathrm{b}$ ).
After 1-week in vitro culture, primary rat neurons formed mature synapses and were ready for oxyhemoglobin $(\mathrm{OxyHb})$ treatment. We observed that miR-21-5p accumulated markedly in conditioned medium from OxyHb- 


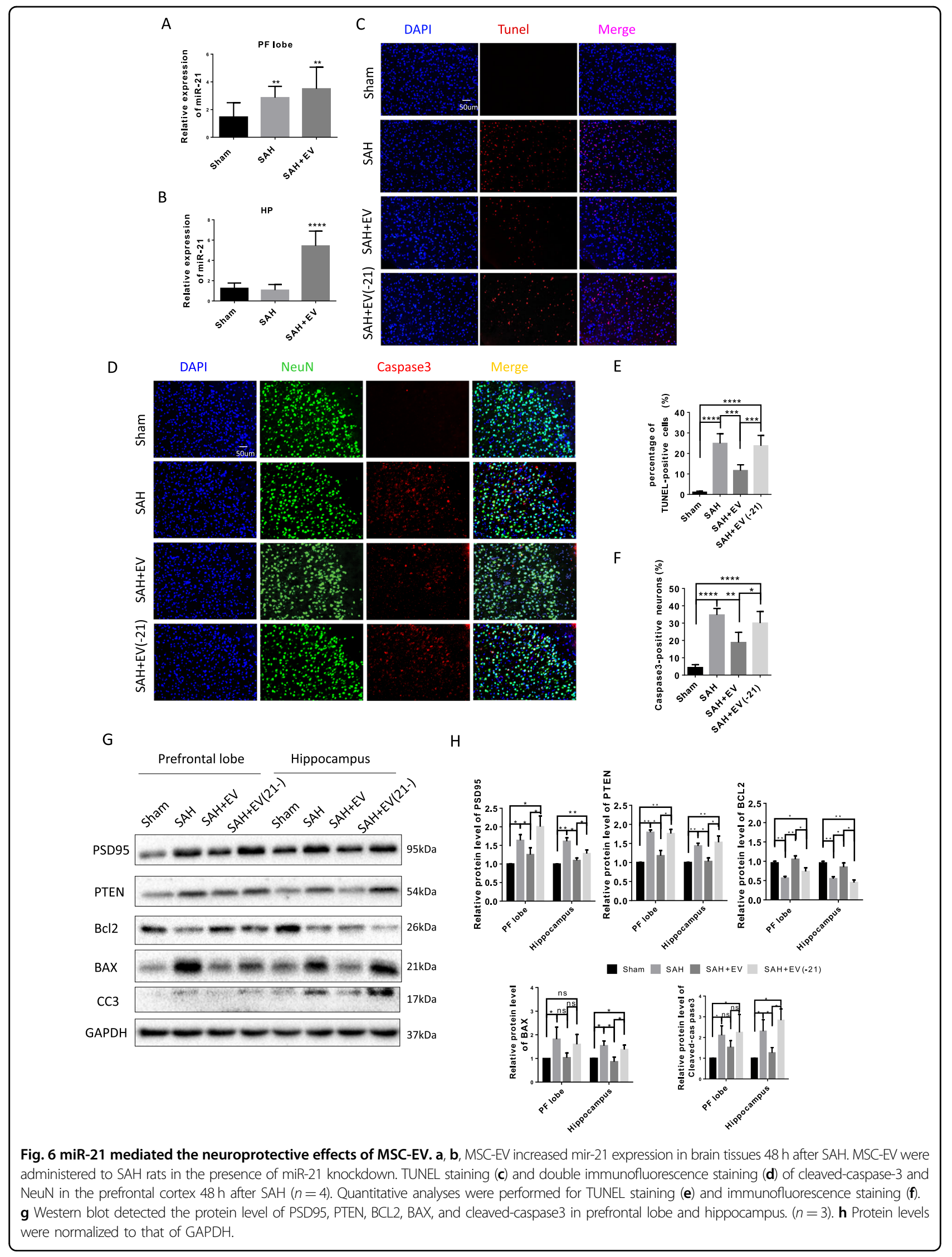




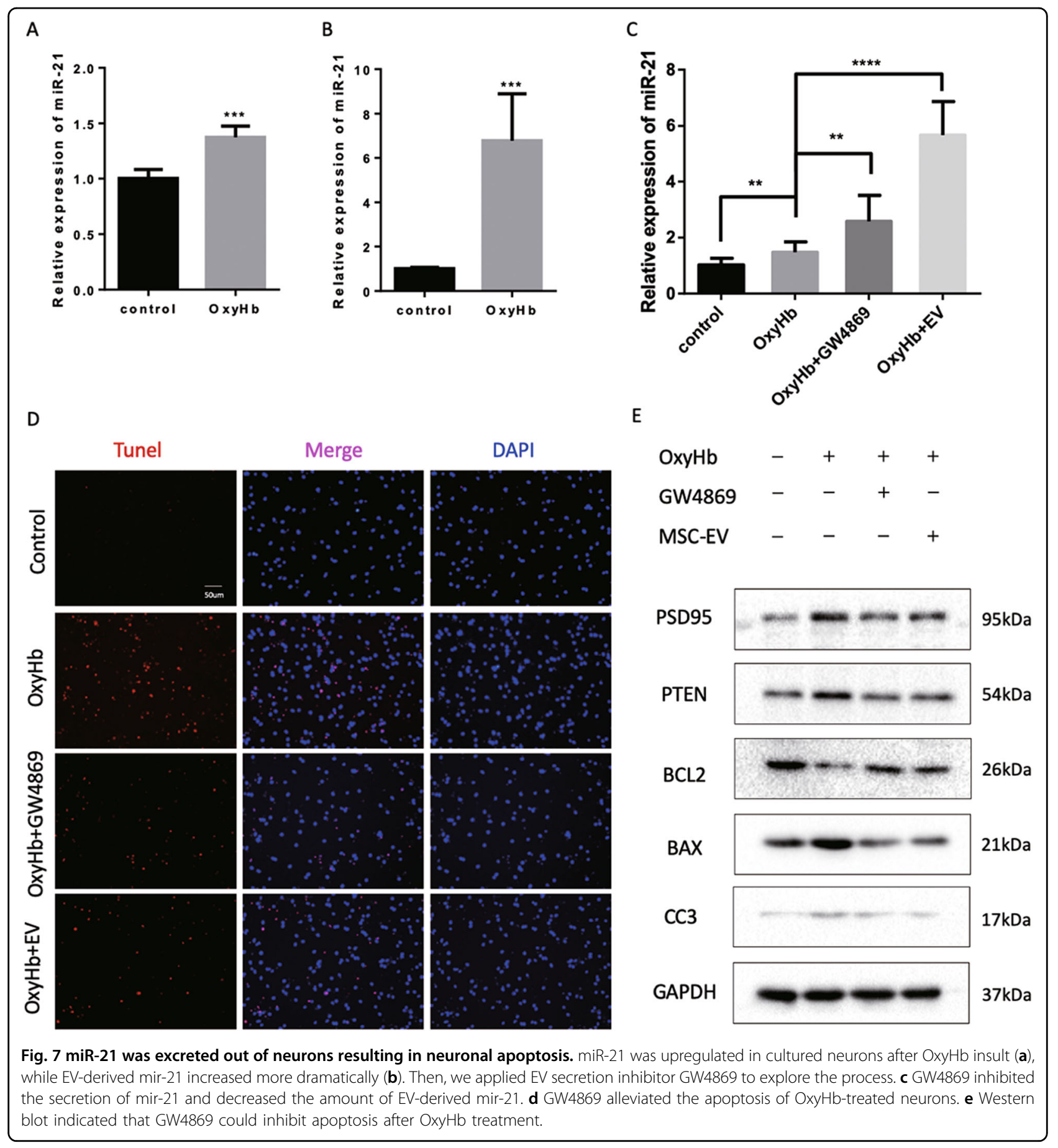

treated $(10 \mu \mathrm{mol} / \mathrm{L})$ neurons than control group. We further employed GW4869 to inhibit EV secretion.

Administration of GW4869 increased the expression of miR-21-5p in neuronal cytoplasm (Fig. 7c) and inhibited neuronal apoptosis resulted from $\mathrm{OxyHb}$ treatment (Fig. 7d). As shown in Fig. 7e, western blotting also supported the anti-apoptosis effects of inhibiting EV secretion. Compared with MSC-EV, GW4869 treatment did not increase miR-21-5p that greatly but also inhibited neuronal apoptosis similarly. However, administration of GW4869 in vivo lack of targeting capacity and will disrupt many other EVmediated beneficial biological activities, which limits its use in SAH-related cognitive dysfunction. 


\section{MiR-21-5p targeted the PTEN/Akt axis in neurons to alleviate $\mathrm{EBI}$ after SAH}

To further investigate the downstream mechanisms underlying the effects of EV-derived miR-21-5p, we used MK2206 to block AKT activation. MK2206 was administered at $100 \mu \mathrm{g}$ per rat dissolved in DMSO through stereotaxic injection into lateral ventricle 2 days ahead of carotid perforation. In the presence of MK2206, MSC-EV could not effectively suppress brain injury and neuronal apoptosis induced by SAH (Fig. 8c-e). Furthermore, a modified Garcia scores test was performed to examine neurological function. As shown in Fig. $8 \mathrm{a}$ and b, both miR-21-5p inhibitors or MK2206 could block the neuroprotective effects of MSC-EV.

\section{Discussion}

Many aneurysmal SAH patients suffer from cognitive function impairments even though they received proper treatment, such as the clipping or coiling of aneurysms, which causes problems in returning to work and burdens the family ${ }^{3,35}$. The severity of bleeding is associated with neuropsychological outcomes ${ }^{36}$. With anti-vasospasm therapy failing to improve outcomes in SAH patients, EBI that occurs in the first $72 \mathrm{~h}$ post SAH has drawn increasing attention in recent years and has emerged as an important risk factor for poor outcomes in $\mathrm{SAH}^{7,37,38}$. EBI is believed to be involved in pathophysiological changes post $\mathrm{SAH}$, including global cerebral edema, ultraearly vasospasm, and reactive neuroinflammation ${ }^{39}$. Thus, EBI may be the root cause of cognitive dysfunction post SAH and needs to be addressed to improve the outcomes of SAH patients.

MSC transplantation has been used to treat rats suffering from $\mathrm{SAH}^{17,18,40,41}$. The therapeutic potential of MSCs in rodent models of SAH was documented for the first time in 2012. Khalili et al. demonstrated that the intravenous administration of MSC to rats that had been subjected to SAH improved the structural integrity of cerebral tissue to promote functional recovery ${ }^{40,41}$. In another study, bone marrow MSC (BMSC) administration alleviated SAH-induced EBI and ameliorated neurobehavioral impairments partly by inhibiting microglia activation $^{17}$, which suggested that there is another mechanism that accounts for this functional protection. However, there are potential risks for using cell-based therapy, like developing tumors or immuno-rejection ${ }^{42,43}$.

Meanwhile, some studies showed that the therapeutic effects of MSC therapy were mediated by EVs secreted by MSCs rather than transdifferentiated $\mathrm{MSCs}^{15,44}$. In addition, the administration of MSC-derived EVs has comparable treatment effects as that of MSCs in rodent models of ischemic stroke and $\mathrm{TBI}^{45}$. Therefore, we hypothesized that MSC therapy might alleviate brain damage in SAH by secreting EVs loaded with biologically functional cargoes and that intravenous MSC-EV administration could recapitulate the response to therapy with the secreting cells.

A few studies have reported miRNA changes after $\mathrm{SAH}^{19,46}$, but its regulatory role remain largely unclear. We demonstrated that protective miR-21-5p increased reactively in neurons upon $\mathrm{SAH}$ induction and wore down through EV secretion, which led to neuronal apoptosis. Moreover, inhibiting EV secretion can restrain more miR-21-5p in neurons. However, GW4869 administration in vivo will disrupt many other benneficial cellto-cell communications and may produce side effects. Fortunately, according to many researches, MSC-EV could target injured neurons to deliver abundant miRNA. Thus, we employed MSC-EV enriched with miR21-5p to replenish miR-21-5p in SAH-insulted neurons. Both in vivo and in vitro, MSC-EV successfully increased miR-21-5p in brain or neurons and protected more neurons form apoptosis, and then improve the cognitive function of SAH rats.

Pten/Akt signaling has been implicated in SAH pathogenesis. S473 Phosphorylation of Akt can protect neuron from death ${ }^{47}$, while the upstream regulator PTEN inhibited neuronal survival ${ }^{28,48}$. In this study, we demonstrated that PTEN was upregulated in OxyHb-treated neurons and prefrontal cortex of SAH-insulted rats and EVderived miR-21-5p from mesenchymal stem cells could significantly inhibited PTEN expression to protect neuronal apoptosis and improve cognitive function.

We employed EV sequencing to identify cargoes the EVs carried and found that miR-21-5p, along with the other top 6 miRNAs, constitutes nearly $60 \%$ of all miRNA contents. miR-21-5p alone accounts for as much as $22.5 \%$ of miRNA contents. Interestingly, an increase in miR-21$5 \mathrm{p}$ was also found in CSF from SAH patients and was correlated with DCI occurrence ${ }^{19}$. As is well known, the PTEN/Akt signaling pathway is regulated by miR-21-5p and promotes cell survival in various diseases, including $\mathrm{SAH}^{49-51}$. Phospho473-Akt levels decreased significantly during EBI after $\mathrm{SAH}^{52,53}$. However, Hidenori et al. reported that Akt phosphorylation was accelerated in cortical and hippocampal neurons after SAH, especially in the first $6 \mathrm{~h}$. However, at $24 \mathrm{~h}$ post SAH, Akt phosphorylation decreased significantly ${ }^{47}$. Furthermore, both previous studies and our preliminary study ${ }^{23}$ showed that the overall protein level of PTEN increased and the pharmaceutical inhibition of PTEN could protect against EBI after $\mathrm{SAH}^{28,54}$. Thus, we proposed that Akt signaling was activated in response to $\mathrm{SAH}$ onset, but that its activity could not be sustained to protect neurons and that miR21-5p as an upstream regulator may be involved in the process. Further studies could reveal the detailed mechanisms involved in $\mathrm{SAH}$ pathogenesis to develop new therapies based on the miR-21-5p/PTEN/Akt 


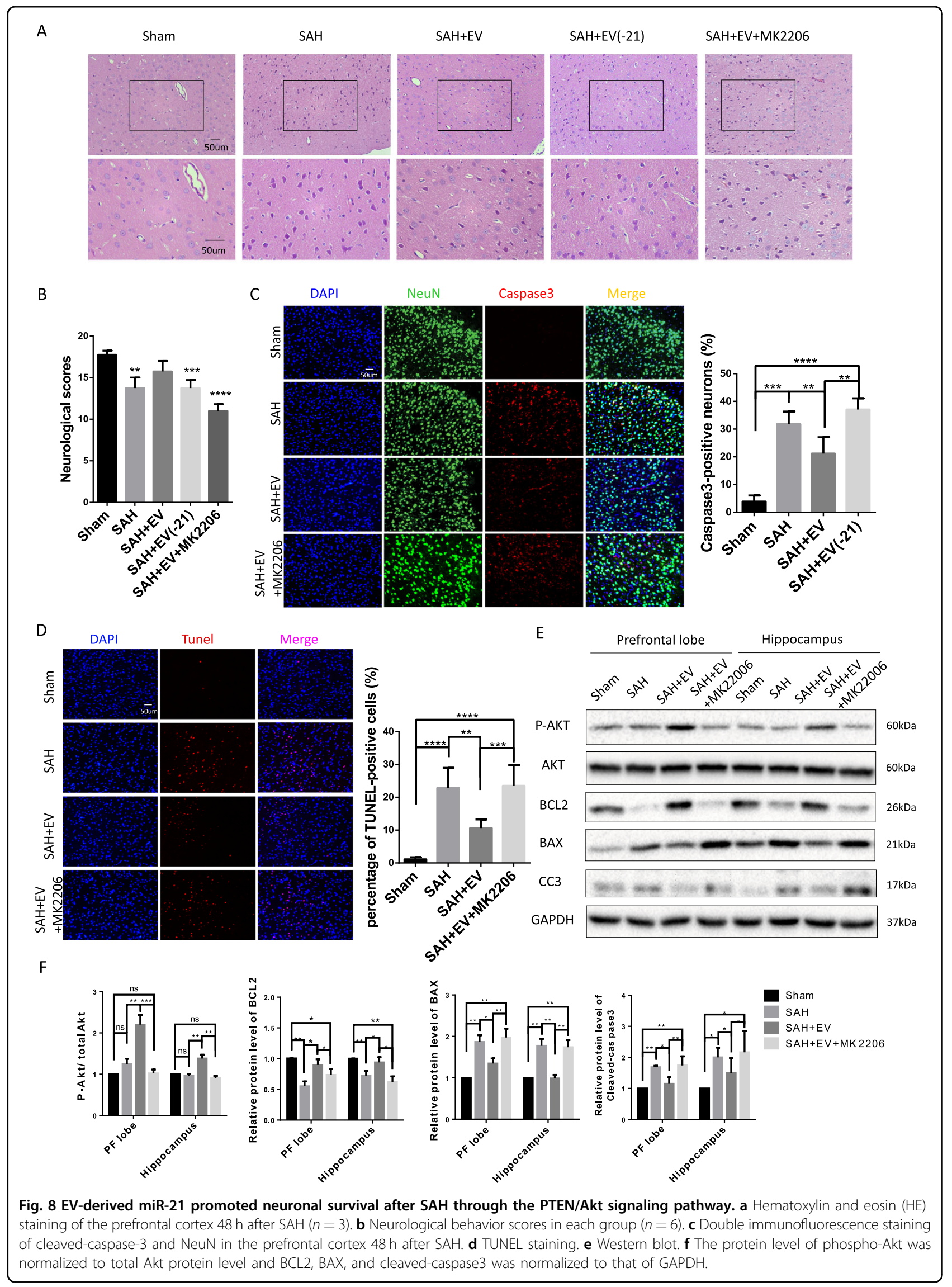


signaling pathway. Using qRT-PCR, we observed that the expression level of miR-21-5p in the prefrontal cortex and hippocampus first increased after SAH yet decreased afterward, implying that miR-21-5p in CSF may originate from neurons and interrupting the discharge of miR-21$5 p$ or replenishing miR-21-5p in neurons may help protect neurons against SAH-induced EBI via PTEN/Akt signaling.

In this study, we identified the protective role of MSCderived EVs in a rat model of SAH. MSC-EV improved neurological scores significantly and alleviated brain edema resulting from SAH. Using EV tracing, we verified that MSC-EV was taken up by damaged neurons to exert its effects. In addition, a TUNEL assay and cleaved-caspase-3/NeuN double staining showed a decrease in apoptosis in the $\mathrm{SAH}+\mathrm{EV}$ group compared with the $\mathrm{SAH}+\mathrm{PBS}$ group, which was also confirmed by Western blot. MSC-EV increased Bcl-2 expression and inhibited Bax and Caspase- 3 expressions in both prefrontal lobe and hippocampus, suggesting that it suppressed cellular apoptosis through inhibiting the mitochondrial apoptosis pathway. To investigate the mechanism involved in the neuroprotective effects of MSC-EV in SAH, we employed miRNA EV sequencing to identify the specific molecular cargo. According to the online data from exRNA Atlas, we assumed that miR-21-5p is the key target that mediates the therapeutic effects.

Thus, miR-21-5p inhibitors and MK2206 were used to explore the involved mechanism, either of which was sufficient to impair the effects of MSC-EV. Furthermore, we showed that SAH rats treated with MSC-EV exhibited better cognitive function in the Morris Water Maze test than those treated with PBS. In brief, we demonstrated that EV-derived miR-21-5p alleviated brain injury after SAH through inhibiting neuronal apoptosis and improved the neurological behaviors via the PTEN/Akt pathway, which provides support for a potential new restorative therapy for SAH patients at risk for cognitive defects.

The current study possesses some limitations and could be improved in several aspects. First, EVs can be decorated with targeting peptides to promote delivery efficiency. For example, rabies virus glycoprotein (RVG) peptide can be fused to the EV-derived membrane protein Lamp2b to enhance targeting capability in the brain ${ }^{55}$. In subsequent studies, RVG EVs were proven to be effective for miRNA or siRNA delivery to the brain for disease treatment ${ }^{56,57}$. In some studies, naive EVs were loaded with additional therapeutic agents to achieve better therapeutic effects. For example, EVs loaded with curcumin or cucurbitacin I suppressed inflammatory responses in models of lipopolysaccharide (LPS)-induced brain inflammation, experimental autoimmune encephalitis, and GL26 brain tumors, respectively ${ }^{58}$. Furthermore, the therapeutic potential of EVs carrying excess miRNA has been investigated. In a rat model of middle cerebral artery occlusion, miR-133b-rich EVs promoted neurite remodeling and functional recovery ${ }^{15,16}$. In a subsequent study, they also demonstrated that engineered MSC EVs containing elevated miR-17-92 clusters had therapeutic benefits for stroke ${ }^{44}$.

However, most studies mentioned above did not employ antibodies of classic EV markers, such as CD63 or CD81, to isolate EVs, which leads to the isolation of a mixture of microvesicles shed from the plasma membrane, endosome-derived real EVs and nonvesicular extracellular nanoparticles ${ }^{59}$. The molecular constituents of these extracellular components differ greatly from each other. Further exploration may help to identify the specific contents that produce therapeutic benefits in animal models of various diseases.

In conclusion, we found that MSC-EV alleviated brain injury after $\mathrm{SAH}$ through inhibiting neuronal apoptosis and improved the neurological behavior, which expands the previous understanding of MSC-based therapy for $\mathrm{SAH}$ and provides evidence for a potential new restorative therapy that could be used for SAH patients at risk of cognitive defects.

\section{Acknowledgements \\ This work was supported by grants from National Natural Science Foundation of China (Nos. 30872645; 81101594; 81372719; 81172403;81300510; 81402077; 81571284; 91542115; 81702468; 81874083;81873768;81802966), Natural Science Foundation of Shandong Province of China (Nos.2017CXGC1203; 2017G006012; ZR2019BH057;2013GGE27006) and Taishan Scholars of Shandong Province of China (No. ts201511093).}

\section{Author details}

'Department of Neurosurgery, Qilu Hospital, Cheeloo College of Medicine, Shandong University, 107 Wenhua Xi Road, Jinan, Shandong Province, China. ${ }^{2}$ Institute of Brain and Brain-Inspired Science, Shandong University, 44 Wenhua Xi Road, Jinan, Shandong Province 250012, P.R. China. ${ }^{3}$ Shandong Provincial Key Laboratory of Brain Function Remodeling, Jinan, Shandong Province, China. ${ }^{4}$ Department of Neurosurgery, The First Affiliated Hospital of Wenzhou Medical University, Wenzhou, Zhejiang Province, China. ${ }^{5}$ Department of Physiology, Shandong University School of Basic Medical Sciences, Jinan, China

\section{Data availability}

Because some of this research is still in progress, the raw data cannot be shared at present.

Conflict of interest

The authors declare that they have no conflict of interest.

\section{Publisher's note}

Springer Nature remains neutral with regard to jurisdictional claims in published maps and institutional affiliations.

Supplementary Information accompanies this paper at (https://doi.org/ 10.1038/s41419-020-2530-0).

Received: 10 November 2019 Revised: 20 April 2020 Accepted: 21 April 2020

Published online: 13 May 2020 


\section{References}

1. de Rooij, N. K., Linn, F. H., van der Plas, J. A., Algra, A. \& Rinkel, G. J. Incidence of subarachnoid haemorrhage: a systematic review with emphasis on region, age, gender and time trends. J. Neurol., Neurosurg. Psychiatry 78, 1365-1372 (2007).

2. Macdonald, R. L. \& Schweizer, T. A. Spontaneous subarachnoid haemorrhage. Lancet 389, 655-666 (2017).

3. Al-Khindi, T., Macdonald, R. L. \& Schweizer, T. A. Cognitive and functional outcome after aneurysmal subarachnoid hemorrhage. Stroke 41, e519-e536 (2010).

4. Hadjivassiliou, M. et al. Aneurysmal SAH: cognitive outcome and structural damage after clipping or coiling. Neurology 56, 1672-1677 (2001).

5. Mayer, S. A. et al. Global and domain-specific cognitive impairment and outcome after subarachnoid hemorrhage. Neurology 59, 1750-1758 (2002).

6. Suzuki, H. What is early brain injury? Transl. Stroke Res. 6, 1-3 (2015)

7. Fujii, M. et al. Early brain injury, an evolving frontier in subarachnoid hemorrhage research. Transl. Stroke Res. 4, 432-446 (2013).

8. Cahill, W. J., Calvert, J. H. \& Zhang, J. H. Mechanisms of Early Brain Injury after Subarachnoid Hemorrhage. J. Cereb. Blood Flow. Metab. 26, 1341-1353 (2006).

9. Kourembanas, S. Exosomes: vehicles of intercellular signaling, biomarkers, and vectors of cell therapy. Annu. Rev. Physiol. 77, 13-27 (2015).

10. Lou, G., Chen, Z., Zheng, M. \& Liu, Y. Mesenchymal stem cell-derived exosomes as a new therapeutic strategy for liver diseases. Exp. Mol. Med. 49, e346 (2017)

11. Tan, C. Y. et al. Mesenchymal stem cell-derived exosomes promote hepatic regeneration in drug-induced liver injury models. Stem Cell Res. Ther. 5, 76 (2014).

12. Aghajani Nargesi, A., Lerman, L. O. \& Eirin, A. Mesenchymal stem cell-derived extracellular vesicles for kidney repair: current status and looming challenges. Stem Cell Res. Ther. 8, 273 (2017).

13. Teng, $\mathbf{X}$. et al. Mesenchymal stem cell-derived exosomes improve the microenvironment of infarcted myocardium contributing to angiogenesis and anti-inflammation. Cell. Physiol. Biochem. 37, 2415-2424 (2015).

14. Zhang, B. et al. HucMSC-exosome mediated-Wnt4 signaling is required for cutaneous wound healing. Stem Cells 33, 2158-2168 (2015)

15. Xin, $\mathrm{H}$. et al. Exosome-mediated transfer of miR-133b from multipotent mesenchymal stromal cells to neural cells contributes to neurite outgrowth. Stem Cells 30, 1556-1564 (2012).

16. Xin, $\mathrm{H}$. et al. MiR-133b promotes neural plasticity and functional recovery after treatment of stroke with multipotent mesenchymal stromal cells in rats via transfer of exosome-enriched extracellular particles. Stem Cells 31, 2737-2746 (2013).

17. Liu, W. et al. Mesenchymal stem cells alleviate the early brain injury of subarachnoid hemorrhage partly by suppression of Notch1-dependent neuroinflammation: involvement of Botch. J. Neuroinflammation 16, 8 (2019).

18. Nijboer, C. H. et al. Intranasal stem cell treatment as a novel therapy for subarachnoid hemorrhage. Stem Cells Dev. 27, 313-325 (2018).

19. Bache, S. et al. MicroRNA changes in cerebrospinal fluid after subarachnoid hemorrhage. Stroke 48, 2391-2398 (2017).

20. Bang, C. et al. Cardiac fibroblast-derived microRNA passenger strand-enriched exosomes mediate cardiomyocyte hypertrophy. J. Clin. Invest 124, 2136-2146 (2014).

21. Sehba, F. A. Rat endovascular perforation model. Transl. Stroke Res. 5, 660-668 (2014).

22. Marbacher, S. Animal models for the study of subarachnoid hemorrhage: are we moving towards increased standardization? Transl. Stroke Res. 7, 1-2 (2016).

23. Li, T. et al. Neuroprotective effects of hydrogen sulfide against early brain injury and secondary cognitive deficits following subarachnoid hemorrhage. Brain Pathol. 27, 51-63 (2017).

24. Garcia, J. H., Wagner, S., Liu, K. F. \& Hu, X. J. Neurological deficit and extent of neuronal necrosis attributable to middle cerebral artery occlusion in rats. Statistical validation. Stroke 26, 627-634 (1995). discussion 635.

25. Hasegawa, Y., Suzuki, H., Altay, O. \& Zhang, J. H. Preservation of tropomyosinrelated kinase $B(T r k B)$ signaling by sodium orthovanadate attenuates early brain injury after subarachnoid hemorrhage in rats. Stroke 42, 477-483 (2011)

26. Sugawara, T., Ayer, R., Jadhav, V. \& Zhang, J. H. A new grading system evaluating bleeding scale in filament perforation subarachnoid hemorrhage rat model. J. Neurosci. Methods 167, 327-334 (2008).

27. Ayer, R. E., Sugawara, T., Chen, W., Tong, W. \& Zhang, J. H. Melatonin decreases mortality following severe subarachnoid hemorrhage. J. Pineal Res. 44 197-204 (2008)
28. Chen, Y. et al. Administration of a PTEN inhibitor BPV(pic) attenuates early brain injury via modulating AMPA receptor subunits after subarachnoid hemorrhage in rats. Neurosci. Lett. 588, 131-136 (2015).

29. Ainsztein, A. M. et al. The NIH extracellular RNA communication consortium. J. Extracell. Vesicles 4, 27493 (2015).

30. Wang, C., Ji, B., Cheng, B., Chen, J. \& Bai, B. Neuroprotection of microRNA in neurological disorders (Review). Biomed. Rep. 2, 611-619 (2014).

31. Yan, $\mathrm{H}$. et al. Long non-coding RNA MEG3 functions as a competing endogenous RNA to regulate ischemic neuronal death by targeting miR-21/ PDCD4 signaling pathway. Cell Death Dis. 8, 3211 (2017).

32. Ge, X. T. et al. miR-21 improves the neurological outcome after traumatic brain injury in rats. Sci. Rep. 4, 6718 (2014).

33. Slota, J. A. \& Booth, S. A. MicroRNAs in neuroinflammation: implications in disease pathogenesis, biomarker discovery and therapeutic applications. Noncoding RNA https://doi.org/10.3390/ncrna5020035 (2019).

34. Cui, G. H. et al. Exosomes derived from hypoxia-preconditioned mesenchymal stromal cells ameliorate cognitive decline by rescuing synaptic dysfunction and regulating inflammatory responses in APP/PS1 mice. FASEB J. 32, 654-668 (2018).

35. Wallmark, S., Ronne-Engstrom, E. \& Lundstrom, E. Predicting return to work after subarachnoid hemorrhage using the montreal cognitive assessment (MoCA). Acta Neurochir. 158, 233-239 (2016).

36. Haug, T. et al. Cognitive outcome after aneurysmal subarachnoid hemorrhage: time course of recovery and relationship to clinical, radiological, and management parameters. Neurosurgery 60, 649-656 (2007). discussion 656-647.

37. Sehba, F. A., Hou, J., Pluta, R. M. \& Zhang, J. H. The importance of early brain injury after subarachnoid hemorrhage. Prog. Neurobiol. 97, 14-37 (2012).

38. Caner, B., Hou, J., Altay, O., Fuj, M. \& Zhang, J. H. Transition of research focus from vasospasm to early brain injury after subarachnoid hemorrhage. J. Neurochemistry 123, 12-21 (2012).

39. Al-Mufti, F. et al. Emerging markers of early brain injury and delayed cerebra ischemia in aneurysmal subarachnoid hemorrhage. World Neurosurg. 107 148-159 (2017)

40. Khalili, M. A et al. Mesenchymal stem cells improved the ultrastructural morphology of cerebral tissues after subarachnoid hemorrhage in rats. Exp. Neurobiol. 23, 77-85 (2014).

41. Khalili, M. A. et al. Therapeutic benefit of intravenous transplantation of mesenchymal stem cells after experimental subarachnoid hemorrhage in rats. J. Stroke Cerebrovasc. Dis. 21, 445-451 (2012).

42. Goldman, S. A. Stem and Progenitor Cell-Based Therapy of the Central Nervous System: Hopes, Hype, and Wishful Thinking. Cell Stem Cell 18, 174-188 (2016).

43. Lee, A. S., Tang, C., Rao, M. S., Weissman, I. L. \& Wu, J. C. Tumorigenicity as a clinical hurdle for pluripotent stem cell therapies. Nat. Med. 19, 998-1004 (2013).

44. Xin, $H$. et al. MicroRNA cluster miR-17-92 cluster in exosomes enhance neuroplasticity and functional recovery after stroke in rats. Stroke $\mathbf{4 8}, \mathbf{7 4 7 - 7 5 3}$ (2017).

45. Zhang, Z. G., Buller, B. \& Chopp, M. Exosomes-beyond stem cells for restorative therapy in stroke and neurological injury. Nat. Rev. Neurol. https:// doi.org/10.1038/s41582-018-0126-4 (2019)

46. Stylli, S. S. et al. miRNA expression profiling of cerebrospinal fluid in patients with aneurysmal subarachnoid hemorrhage. J. Neurosurg. 126, 1131-1139 (2017).

47. Endo, H., Nito, C., Kamada, H., Yu, F. \& Chan, P. H. Akt/GSK3beta survival signaling is involved in acute brain injury after subarachnoid hemorrhage in rats. Stroke 37, 2140-2146 (2006)

48. Qin, X. et al. MicroRNA-26b/PTEN signaling pathway mediates glycine-induced neuroprotection in SAH injury. Neuro. Res. https://doi.org/10.1007/s11064-01902886-2 (2019).

49. Panagal, M. et al. Dissecting the role of miR-21 in different types of stroke. Gene 681, 69-72 (2019)

50. Krichevsky, A. M. \& Gabriely, G. miR-21: a small multi-faceted RNA. J. Cell. Mol. Med. 13, 39-53 (2009)

51. $\mathrm{Xu}, \mathrm{X}$. et al. miR-21 in ischemia/reperfusion injury: a double-edged sword? Physiol. Genomics 46, 789-797 (2014).

52. Duris, K. et al. alpha7 nicotinic acetylcholine receptor agonist PNU-282987 attenuates early brain injury in a perforation model of subarachnoid hemorrhage in rats. Stroke 42, 3530-3536 (2011). 
53. Topkoru, B. C. et al. Nasal administration of recombinant osteopontin attenuates early brain injury after subarachnoid hemorrhage. Stroke 44, 3189-3194 (2013).

54. Hasegawa, Y., Suzuki, H., Altay, O., Chen, H. \& Zhang, J. H. Treatment with sodium orthovanadate reduces blood-brain barrier disruption via phosphatase and tensin homolog deleted on chromosome 10 (PTEN) phosphorylation in experimental subarachnoid hemorrhage. J. Neurosci. Res. 90, 691-697 (2012).

55. Alvarez-Erviti, L. et al. Delivery of siRNA to the mouse brain by systemic injection of targeted exosomes. Nat. Biotechnol. 29, 341-345 (2011).
56. Yang, J., Zhang, X., Chen, X., Wang, L. \& Yang, G. Exosome mediated delivery of miR-124 promotes neurogenesis after ischemia. Mol. Ther. Nucleic Acids 7 , 278-287 (2017)

57. Liu, Y. et al. Targeted exosome-mediated delivery of opioid receptor Mu siRNA for the treatment of morphine relapse. Sci. Rep. 5, 17543 (2015).

58. Zhuang, $X$. et al. Treatment of brain inflammatory diseases by delivering exosome encapsulated anti-inflammatory drugs from the nasal region to the brain. Mol. Ther. 19, 1769-1779 (2011)

59. Jeppesen, D. K. et al. Reassessment of exosome composition. Cell 177 428-445.e418 (2019). 